\title{
A Framework to Include Wind-Thermal Bundled Power Transmission Pattern in Multi-region Generation Expansion Planning Model
}

\author{
Shengyu Wu and Xufeng Song
}

\begin{abstract}
Based on duration curve method, a framework to include wind-thermal bundled power transmission pattern in multi-region generation expansion planning model is proposed in this paper. This framework proposes modelling for receiving end region, sending end region and transmission side, handling two patterns of wind-thermal bundled power transmission, fixed ancillary thermal generators and non-fixed ancillary thermal generators.
\end{abstract}

Index Terms - Wind-thermal bundled power transmission, multi-region generation expansion planning, duration curve.

\section{INTRODUCTION}

Wind power and PV power are promising renewable resources and the penetration level will further increase in the future. It is inevitable to involve renewable energy in long-term Multi-Region Generation Expansion Planning (MR-GEP) [1]. In long-term MR-GEP, the objective is to expand the existing power system to serve the forecasted load over a planning horizon of typically 10-30 years. Load duration curve has been widely used as basis for relevant further optimization in MR-GEP [2], [3].

In essence, long-term MR-GEP is the expansion optimization of dispatchable generations. The non-dispatchable generation modelling of renewable energy is often reflected on the load side. To include the uncertainty characteristic of the non-dispatchable renewable generation, there are commonly two ways. One is at the fixed load level, the probability distribution of renewable production is taken into consideration [4]-[9]. The second way to handle non-dispatchable generation is to consider the joint distribution with load. Ref. [9] breaks down the joint distribution into several cells; and sequential approximation is introduced to reduce computational effort. These methods, however, only reflect load amount, and neglect the time dependence of load, which further has high correlation with the output of PV and wind generation, obviously, PV generates only during day time. Besides, the simplicity of LDC is lost in these methods.

Traditionally, as thermal power dominate the power system, MR-GEP treat transmission as dispatchable. However, under large-scale renewable energy transmission circumstances, the transmission is becoming

Manuscript received November 24, 2015; revised February 13, 2016

Shengyu Wu is with the Department of Energy Strategy and Planning State Grid Energy Research Institute, Beijing, China (e-mail: wushengyu@sgeri.sgcc.com.cn).

Xufeng Song is with the State Grid Xinyuan Company Ltd, Beijing, China (e-mail: songxf@sgxy.sgcc.com.cn). non-dispatchable for the sending end region. Though there are many researches in non-dispatchable generation modelling in MR-GEP, how to model the non- dispatchable transmission in MR-GEP remains an open issue. In China, renewable energy and thermal power distribution areas overlap, wind-thermal bundled power transmission is preferred.

Based on duration curve method, a framework to include wind-thermal bundled power transmission pattern in multi-region generation expansion planning model is proposed in this paper. This framework handles two patterns of wind-thermal bundled power transmission, fixed ancillary thermal generator and non-fixed ancillary thermal generator. The remainder of this paper is structured as follows. In Section II, two patterns of wind-thermal bundled power transmission and transmission duration curve is briefly discussed. Residual load duration curve with renewable transmission in the receiving end region is presented in Section III. Section IV discusses sending end region and transmission modelling. Section V summaries the whole framework with flowchart. Section VI presents the conclusion.

\section{WIND-THERMAL BUNDLED POWER TRANSMISSION PATTERN}

Ten-million kilowatt renewable energy bases China plans to build are mainly distributed in the western, northern and eastern coastal areas, coal power bases are mainly concentrated in the western and northern regions. Renewable energy and thermal power distribution areas overlap, and there are objective needs for these two kinds of power to transmit large-scale, long-distance to eastern and central load centers in China. Bundling renewable energy and thermal power with $\mathrm{AC}$ and $\mathrm{DC}$ outgoing manner, not only meet the basic requirements for large-scale conventional power and renewable energy delivery, and can ensure a smooth transmission pattern, a substantial increase in the utilization of the transmission channel.

Depending on the AC or DC transmission manner, the bundled coal power generation could be divided into two types. If DC transmission is adopted, the bundled coal-fired power come from the specific coal-fired generators near the renewable energy base, it is called fixed ancillary thermal generator type in this paper. If $\mathrm{AC}$ transmission is adopted, the bundled coal-fired power come from the nearby coal-fired generators as well as other generators on the grid, it is called non-fixed ancillary thermal generator type in this paper. These two types induce two patterns of modelling in 
the transmission sending region, which will be discussed in the later part.

\section{A. Renewable Energy Duration Curve and Simplification}

Renewable energy duration curve refers to the yearly duration curve of accumulated output of wind power, solar power, hydropower and other renewable energy generators which are aimed to be transmitted by a certain transmission channel. Renewable energy duration curve describes the cumulative number of hours the accumulated outputs exceeds a certain value in one year.

A linear line $l(w)$ is used to approximate the renewable energy duration curve, where $w \in[0,8760]$. The uppermost point of the linear line $l(0)=C_{R}$ is the intersection point of extended line cross the maximum output of renewable energy duration curve under a certain guaranteed rate (e.g., 97\%) and vertical axis $w=0$. Portion maximum output of renewable energy duration curve, namely those values greater than the linear line $l(w)$ beyond guaranteed rate is ignored by approximation. This portion of the electricity amount typically account for $2-3 \%$ of the total generating electricity of renewable energy, reflecting the pre-curtailment in order to ensure transmission economy. The ratio of $C_{R}$ to the total installed capacity of renewable energy is denoted as renewable energy transmission capacity factor, $\varepsilon=C_{R} /\left(C_{W}+\right.$ $C_{S}+C_{H}$ ), where $C_{W}, C_{S}$ and $C_{H}$ denote the installed capacity of wind farms, solar power and hydro power, $\varepsilon<1$.

\section{B. Wind-Thermal Bundled Power Transmission Duration Curve}

The main role for bundled coal power generation is to flatten the chronological transmission curve, since transmission volatility allowance is low in China. For the sake of generation expansion and transmission expansion research, the ancillary service offered by thermal generators is reflected as filling the valley of renewable energy duration curve. Hence, the schematic view of wind-thermal bundled power transmission duration curve is shown in Fig. 1. Area denoted by $E_{1}$ is the renewable electricity curtailment due to transmission capacity limitation. Shadowed area denoted by $E_{2}$ is the transmission amount of renewable electricity, equals the part of renewable energy duration curve excluded by the curtailment. Dotted area denoted by $E_{3}$ is the ancillary service offered by thermal power to decrease transmission volatility and to increase the utilization of transmission channel. Dotted area denoted by $E_{4}$ is the optional electricity by thermal generator to meet the requirement of minimum load ratio for coal-fired plants.

\section{RESIDUAL LOAD DURATION CURVE WITH RENEWABLE TRANSMISSION IN THE RECEIVING END}

Renewable energy and load are uncertainty sources in power system. It is the integrated effects of these uncertainties affecting the reaction of dispatchable generations. For a certain season, during a certain time segment within the day, the outputs of non-dispatchable generation and load would have very low correlation, and thus can be modelled as independent variables. Denote $T_{i}$ as the same certain time interval within a day of the whole season, $i=1, \ldots, n ; T_{i}=\bigcup_{j}\left[\begin{array}{ll}T_{i j}^{1} & T_{i j}^{2}\end{array}\right], j$ is the index of days in the season. Denote the probability distribution, cumulative probability distribution and duration curve of load $x_{i}$ by $f_{T_{i}}(x), F_{T_{i}}(X)$ and $I_{T_{i}}(X)$ respectively, where $I_{T_{i}}(X)=1-F_{T_{i}}(X), \quad X \geq x$. Denote the cumulative distribution of residual load with transmission input when $t \in T_{i}$ by $L_{T_{i}}(Z)$; the cumulative distribution of residual load with transmission input when $t \in T$ by $L(Z)$, where $T=\bigcup_{i} T_{i}$. Then, the residual load curve with transmission input $N(Z)$ becomes

$$
\begin{aligned}
N(Z) & =1-L(Z) \\
& =1-\left(\frac{1}{\tau}\right) \times \sum_{i=1}^{n} \tau_{i} L_{T_{i}}(Z)
\end{aligned}
$$

where $\tau_{i}=\sum_{j} T_{i j}^{2}-T_{i j}^{1}, \tau=\sum_{i} \tau_{i}$.

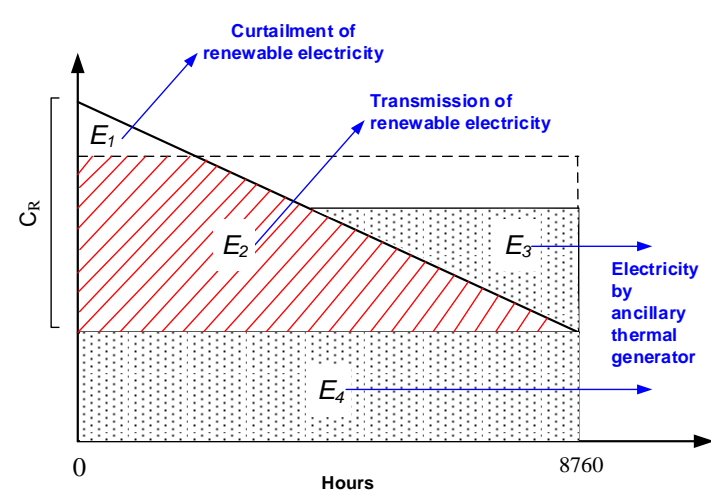

Fig. 1. The schematic view of wind-thermal bundled power transmission duration curve.

With probability distribution of transmission input $g_{T_{i}}(y)$ within time interval $T_{i}$ be written as $g_{T_{i}}\left(y_{k}\right)=p_{T_{i} k}$, where $\sum_{k} p_{T_{i} k}=1$. The residual load cumulative distribution with transmission input $L_{T_{i}}(Z)$ could be written as the following equation.

$$
\begin{aligned}
L_{T_{i}}(Z) & =\iint_{x-y \leq Z} f_{T_{i}}(x) g_{T_{i}}(y) d x d y \\
& =\int_{-\infty}^{+\infty}\left[\int_{-\infty}^{Z+y} f_{T_{i}}(x) g_{T_{i}}(y) d x\right] d y \\
& =\int_{-\infty}^{+\infty} g_{T_{i}}(y) F_{T_{i}}(Z+y) d y \\
& =\sum_{k} p_{T_{i} k} F_{T_{i}}(Z+y)
\end{aligned}
$$

Substitute the above equation, the residual load curve with renewable transmission input $N(Z)$ could be obtained from the sum of sub-intervals' weighted average shifted left.

$$
\begin{aligned}
N(Z) & =1-\left(\frac{1}{\tau}\right) \times \sum_{i=1}^{n} \sum_{k} \tau_{i} p_{T_{i} k} F_{T_{i}}\left(Z+y_{k}\right) \\
& =\left(\frac{1}{\tau}\right) \times \sum_{i=1}^{n} \sum_{k} \tau_{i} p_{T_{i} k} I_{T_{i}}\left(Z+y_{k}\right)
\end{aligned}
$$


Obtaining the probability distribution of transmission input $g_{T_{i}}(y)$ is not a difficult task since the transmission duration curve is settled in the previous section. It should be pointed out that, in the receiving end, if there exist local renewable energy consumption, then $g_{T_{i}}(y)$ should accumulate the effect of local renewable output as well.

\section{Modelling In the SENDING SIDE}

\section{A. Fixed Ancillary Thermal Generator Pattern}

Since the renewable energy for transmission and the fixed ancillary generators are intended to the receiving end only, hence they could be considered as remote generators for receiving end region though their physical location is in the sending end region. They will affect the transmission modelling, not the sending end region modelling.

\section{1) Installed generation capacity for transmission and transmission channel capacity}

As the thermal power plays a main role in power system, the transmission channel capacity equals the installed generation capacity for transmission in generation expansion planning and transmission expansion planning. Take into account the low capacity factor of renewable energy, under large-scale renewable energy transmission circumstances, transmission channel capacity no longer equals the installed generation capacity. For fixed ancillary thermal generator pattern, it is necessary to identify these two capacities.

The installed generation capacity for transmission refers to the total installed capacity for bundled transmission generators or collection bus. In the case of sending side with a variety of energy resources, it usually consists of installed capacity of thermal power, wind power, solar power and so on.

Transmission channel capacity refers to the rated capacity of transmission channel meeting the transmission economy requirement. Under the multi-type power bundled transmission pattern, the transmission channel capacity is often less than the installed generation capacity for transmission.

\section{2) Analytical expression for transmission channel capacity}

With wind-thermal bundled power transmission duration curve analyzed above, constraints including the curtailment proportion of renewable energy must less than the upper limit $\eta_{R}$, as well as thermal power output to meet the economic load rate $\alpha_{C}$ are considered to derive the analytical expression for transmission channel capacity. Boundary conditions including thermal power installed capacity $C_{C}$, minimum output rate of thermal power $\kappa_{C}$ and transmission channel utilization coefficient $\alpha_{C}$. It should be noted that the curtailment proportion of renewable energy does not include the portion considering the renewable energy transmission capacity factor.

The curtailment proportion of renewable energy must less than the upper limit $\eta_{R}$. The portion of renewable energy greater than the difference of transmission channel capacity and minimum thermal output will be curtailed, which is denoted by $E_{1}$ in Fig. 1. Its ratio with generated renewable electricity satisfies:

$$
\left(C_{R}-C_{T}+\kappa_{C} C_{C}\right)^{2} / C_{R}^{2} \leq \eta_{R}
$$

Under transmission channel utilization coefficient $\alpha_{T}$, load rate of thermal power should be larger than its economic load rate $\alpha_{C}$, i.e., transmission electricity subtracted by renewable energy transmission electricity( which is denoted by $E_{3}$ and $E_{4}$ in Fig. 1) is larger than or equal to the electricity generated with economic load rate $\alpha_{C}$ :

$$
\alpha_{T} C_{T}-\frac{1}{2} C_{R}\left[1-\left(C_{R}-C_{T}+\kappa_{C} C_{C}\right)^{2} / C_{R}^{2}\right] \geq \alpha_{C} C_{C}
$$

By the inequalities (1) and (2), relationship between transmission channel capacity $C_{T}$ and installed generation capacity for transmission can the derived as follows.

$$
\begin{aligned}
& C_{r} \geq \max \left\{\kappa_{C} C_{C}+\varepsilon\left(1-\sqrt{\eta_{R}}\right)\left(C_{W}+C_{S}+C_{H}\right), \kappa_{C} C_{C}+\varepsilon\left(1-\alpha_{T}\right)\left(C_{W}\right.\right. \\
& \left.+\sqrt{\varepsilon^{2}\left(1-\alpha_{T}\right)^{2}\left(C_{W}+C_{S}+C_{H}\right)^{2}+2 \varepsilon\left(\alpha_{C}-\kappa_{C} \alpha_{T}\right) C_{C}\left(C_{W}+C_{S}+C_{H}\right)}\right\}
\end{aligned}
$$

Inequality (3) can be added as an additional capacity constraint in transmission channel selection in generation and transmission expansion planning model.

\section{B. Non-Fixed Ancillary Thermal Generator Pattern}

Compared with fixed ancillary thermal generator pattern, there are two differences in non-fixed pattern. Firstly, minimum thermal power output rate for ancillary thermal generator is not an additional constraint in non-fixed pattern. The ancillary service for renewable energy transmission is distributed among dispatchable generators within the sending end region. And for those dispatchable generators, minimum output rates have been already modelled in traditional multi-region generation expansion planning. Transmission channel capacity (1) could be rewritten to:

$$
C_{T} \geq\left(1-\sqrt{\eta_{R}}\right) C_{R}
$$

Secondly, ancillary service for renewable energy transmission should be added as additional load in the sending end region, i.e., load duration curve in the sending end should be added directly with the ancillary service denoted by $E_{3}$.

\section{OVERALL FLOWCHART}

The overall flowchart to include wind-thermal bundled power transmission pattern in multi-region generation expansion planning model is summarized in Fig. 2.

\section{CONCLUSION}

A framework to include wind-thermal bundled power transmission pattern in multi-region generation expansion planning model is proposed, handling two patterns of wind-thermal bundled power transmission - fixed ancillary thermal generators and non-fixed ancillary thermal generators. To model the two different patterns, two concepts are emphatic in this paper, installed generation capacity for transmission and transmission channel capacity. 
Transmission duration curve, residual duration curve based on sub time-segment are the kernel of the simplicity of this framework.

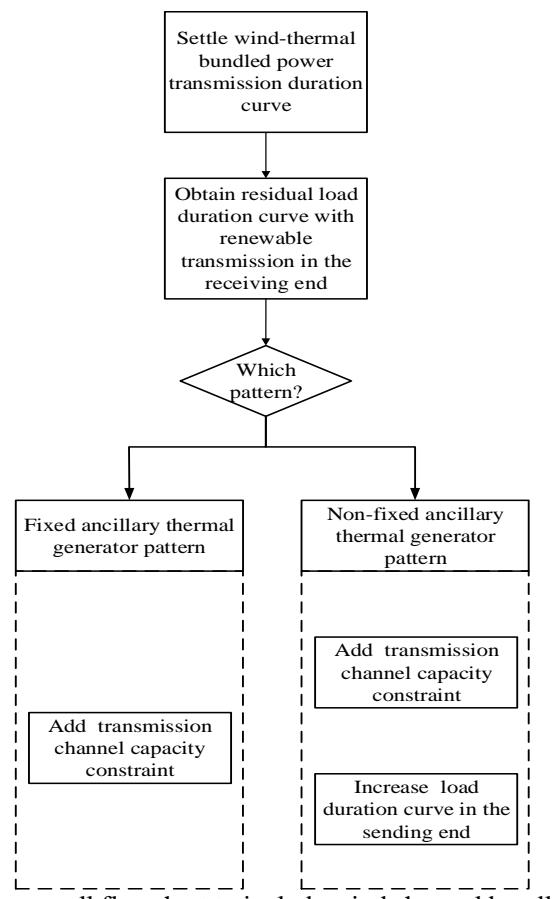

Fig. 2. The overall flowchart to include wind-thermal bundled power transmission pattern in multi-region generation expansion planning.

\section{REFERENCES}

[1] R. Hemmati, R. Hooshmand, and A. Khodabakhshian, "Comprehensive review of generation and transmission expansion planning," Generation, Transmission \& Distribution, vol. 9, pp. 955-964, Jul. 2013

[2] R. R. Booth, "Power system simulation model based on probability analysis," IEEE Transactions, vol. PAS-91, pp. 62-69, 1972.
[3] Y. H. Feng and S. Ryan, "Application of scenario reduction to LDC and risk based generation expansion planning," in Proc. 2012 IEEE Power and Energy Society General Meeting, pp. 1-8.

[4] A. Khan, Y. Y. Sun and A. Ashfaq, "Generation expansion planning considering externalities for large scale integration of renewable energy," in Proc. 2014 IEEE International Conference on Intelligent Energy and Power Systems, pp. 135-140.

[5] C. Zhang, Y. Ding, J. Ostergaard et al., "Generation expansion planning considering integrating large-scale wind generation," in Proc. 2013 39th Annual Conference of the IEEE Industrial Electronics Society, pp. 2051-2056.

[6] O. Mohammed, Y. Amirat, M. Benbouzid et al., "Hybrid generation systems planning expansion forecast: a critical state of the art review," in Proc. 2013 39th Annual Conference of the IEEE Industrial Electronics Society, pp. 1668-1673.

[7] M. S. Javadi, M. Saniei, H. R. Mashhadi et al., "Multi-objective expansion planning approach: distant wind farms and limited energy resources integration," Renewable Power Generation, vol. 6, pp. 652-668, Jul. 2013.

[8] Y. Y. Sun, T. Han, and A. Ashfaq, "A chance-constrained programming based renewable resources included generation expansion planning method and its application," in Proc. 2012 Asia-Pacific Power and Energy Engineering Conference, pp. 1-5.

[9] H. Park and R. Baldick, "Transmission planning under uncertainties of wind and load: sequential approximation approach," IEEE Trans. Power Systems, vol. 28, pp. 2395-2402, Mar. 2013.

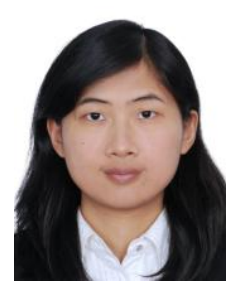

Shengyu Wu was born in Jiangxi province, China. She received her $\mathrm{PhD}$ degree in electrical engineering from Tsinghua University in 2010. Currently, she is with the State Grid Energy Research Institute. Her main research interest is energy and power development strategy and planning.

Xufeng Song is currently with the State Grid Xinyuan Company Ltd.. His main research interest is the operation and planning of pumped storage station in power systems. 


\section{Energy Policy and Biogas}


